\title{
THE TRANSVERSE THERMOMAGNETIC EFFECT IN NICKEL AND COBALT.
}

\author{
By Alpheus W. Smith.
}

$\mathrm{N}$ an earlier paper the author ${ }^{1}$ has studied the Hall effect in iron, nickel and cobalt at a number of temperatures between $-190^{\circ}$ and $I 100^{\circ} \mathrm{C}$. For a given magnetic field the Hall effect in these metals was found to increase more and more rapidly, until the critical temperature of the metal in question was reached, where for a small additional increase in temperature the effect sinks suddenly to a small fraction of its value at that temperature and then continues to decrease with further rise of temperature. In each of these metals it is known that there is a molecular change at the critical temperature. The nickel changes from $\alpha$-nickel to $\beta$-nickel; the iron from $\alpha$-iron to $\beta$-iron and then to $\gamma$-iron; and the cobalt from $\alpha$-cobalt to $\beta$-cobalt. These molecular transformations manifest themselves also in a change of the electrical resistance, ${ }^{2}$ in a change of the thermoelectric heights ${ }^{3}$ and in a change in the magnitude of the Peltier effect. ${ }^{4}$ These latter changes are, however, not large in comparison with the change in the Hall effect or with the change in the permeability. This behavior of the Hall effect at the critical temperature allies it more closely to the magnetic properties of the metal than to either the resistance or the thermoelectric heights. It seemed of interest to examine some of the other effects which are allied to the Hall effect, in order to see in what way they are influenced by changes of temperature and by the molecular transformations which are known to take place when the metals pass from the magnetic to the non-magnetic state.

For a clear statement of the various phenomena which arise when a metal plate which is carrying either a current of heat or a current of electricity is brought into a magnetic field so that the lines of force are normal to the plane of the plate, reference is made to the paper by $\mathrm{H}$. $Z_{\text {Zahn }}^{5}$ and to the paper by Hall and Campbell ${ }^{6}$ on these phenomena. For our present purposes it is only necessary to recall that when a metal

1 Phys. Rev., 30, p. I, Igro.

${ }^{2}$ Harrison, Phil. Mag. (6), 3, p. I83.

3 Ibid., p. I92.

${ }^{4}$ Cermak, Ann. der Phys., 24, p. 35I, I907.

${ }^{5}$ Ann. d. Phys., I4, p. 886, I904.

${ }^{6}$ Proc. Amer. Soc. of Arts and Sci., 46, p. 625, I9rr. 
plate which is carrying a longitudinal electrical current is brought into a magnetic field so that the lines of force are perpendicular to the plane of the plate, there is set up in the plate a transverse electromotive force whose magnitude is given by the equation,

$$
E=R \frac{H i}{d},
$$

where $E$ is the electromotive force in absolute units; $H$, the magnetic field in absolute units; $i$, the current in absolute units; $d$, the thickness of the plate in centimeters.

When a similar plate through which there is a longitudinal flow of heat is brought into a magnetic field in the way indicated above there is set up by the magnetic field a transverse electromotive force given by the equation,

$$
E=Q \beta H \frac{\partial T}{\partial x},
$$

where $E$ is the electromotive force in absolute units; $\beta$, the width of the plate in centimeters; $H$, the magnetic field in absolute units; $\partial T / \partial x$, the temperature gradient in the plate in degrees Centigrade per centimeter; $Q$, a constant which is a function of the temperature of the plate and sometimes of the magnetic field. This phenomenon is known as the transverse thermomagnetic effect or as the Nernst effect. Its variation with the temperature of the plate and its relation to the Hall effect and to the magnetic properties form the subject of the present paper.

Apparatus and Method.

For the study of the transverse thermomagnetic effect between room temperature and $100^{\circ} \mathrm{C}$., the apparatus for securing the desired temperature gradient in the plates, was the same as that used by the author ${ }^{1}$ in the study of this effect in alloys. It consisted essentially of two copper tubes to each of which was soldered a narrow strip of copper about 0.5 $\mathrm{cm}$. in thickness. To these copper strips were soldered the ends of the plates to be investigated. Through one of the tubes flowed steam at atmospheric pressure; through the other tube, water at room temperature. The plates which were about $4 \mathrm{~cm}$. long, $1.5 \mathrm{~cm}$. wide and $0 . \mathrm{I}$ $\mathrm{cm}$. thick, were cut from a larger sheet of the metal so that narrow arms projecting from the middle of each of the longer edges, extended some distance out of the magnetic field. To the ends of these arms which were filed down until they were about $0.1 \mathrm{~cm}$. in diameter were soldered

${ }^{1}$ Phys. Rev., 32, p. I78, I9II. 
the copper lead wires which lead to the galvanometer on which the effect was to be observed. Where the original sheet was not large enough to allow arms of sufficient length to extend out of the magnetic field, the arms were built up out of shorter pieces of the metal by silver-soldering these pieces together. By means of these arms the error due to the transverse galvanomagnetic effect discovered by Leduc ${ }^{1}$ is eliminated, for the junction of the lead wire and the plate can not form a thermal couple since both are of the same material. To determine the temperature gradient in the plate a copper-nickel thermal couple was soldered to either end of the plate on a line midway between its upper and lower edges.

To secure the desired temperature gradients in the plate at temperatures above $100^{\circ} \mathrm{C}$. an electric heating device was constructed. It consisted essentially of two copper $\operatorname{rods} A$ and $B$ (Fig. I $a$ ). These rods

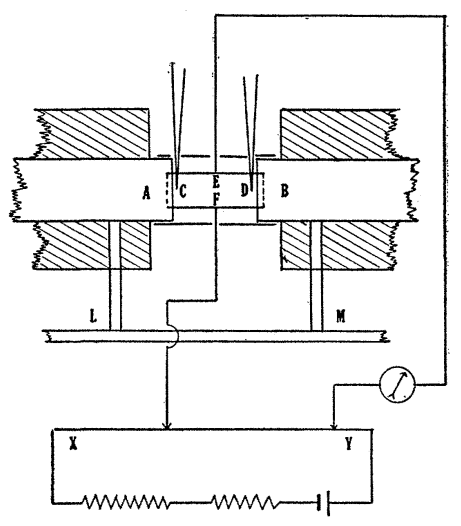

Fig. 1a.

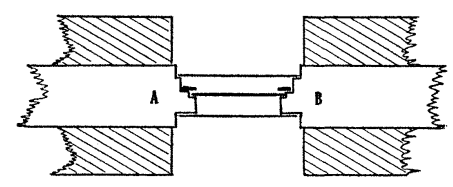

Fig. 1b.

were about $3.5 \mathrm{~cm}$. in diameter and about $13 \mathrm{~cm}$. in length. They were connected by the brass bar $L M$ in such a way that they were electrically insulated from each other. Around each of these rods was wrapped two layers of nichrome wire, insulated from each other and from the rods by means of asbestos and strips of mica. The rods and their windings were then covered with a large number of layers of asbestos paper to afford protection against the loss of heat. The coils of nichrome wire were then connected independently to a suitable source of alternating current. By sending suitable currents through these coils any desired temperature gradient could be obtained and the temperature of the plate could be increased or decreased at will. In order to fasten the ends of ${ }^{1}$ Compt. Rend. I04, p. 1785 (I887). 
the plates to the ends of the rods, the rods were cut away as indicated in Fig. $\mathbf{I} b$ which is a cross section of the rods and plate perpendicular to the plane of the plate. The plate was held in the recess provided for it by means of heavy copper clamps which fastened it firmly to the copper rod. To prevent the lateral flow of heat from the plate and to insure as far as possible a uniform temperature gradient in the plate, it was enclosed in a thin box of non-conducting material. The front and back of the box (Fig. $\mathrm{s} b$ ) were made of thick sheets of mica which were fastened by means of screws to the ends of the copper rods. The top and bottom of the box were made of heavy asbestos board, also fastened by screws to the ends of the copper rods. This box was about $1.8 \mathrm{~cm}$. thick, $4 \mathrm{~cm}$. high, and about $5.5 \mathrm{~cm}$. long. It was filled with powdered magnesium oxide and when the plate was in position there was between the plate and the walls of the box an insulating layer of this magnesium oxide about $0.8 \mathrm{~cm}$. in thickness.

At $C$ and $D$ (Fig. I $a$ ) was clamped a thermal couple to determine the temperature gradient in the plate. For the lower temperatures coppernickel thermal couples were used; for higher temperatures, platinumplatinum-iridium couples. From $E$ and $F$ projected the arms which were cut out of the larger sheet of metal in the manner already described. To these arms were soldered the lead wires which after protection against thermal electromotive forces lead through the galvanometer to the potentiometer $X Y$ on which the difference of potential between $E$ and $F$ was measured in the usual way. There was also in the circuit with the galvanometer an auxiliary potentiometer for compensating the thermal electromotive forces that happened to be in the circuit.

The cobalt and the nickel for one of the nickel plates was obtained from Kahlbaum. The nickel for the other nickel plate was furnished by Eimer and Amend and referred to by them as chemically pure.

The plate was placed between the poles of the magnet in the usual manner and rigidly supported. The currents in the heating coils were then regulated until the desired temperature gradient was secured. When the thermal couples showed that temperature equilibrium had been established, the thermal electromotive forces which happened to be in the galvanometer circuit were as nearly as possible compensated and the difference of potential produced between the edges of the plate by the establishment of the magnetic field was then measured. The direction of the magnetic field having been reversed, the corresponding difference of potential was again measured. The mean of these two observations was taken as the electromotive force called forth by the magnetic field. Sometimes the differences of potential obtained for 
opposite directions of the magnetic field were not equal, because there was some change in the thermal electromotive forces in the reversal of the magnetic field. It was found, however, that the mean of the observations just described eliminated this dissymmetry. These observations were repeated a number of times for each magnetic field and the mean of these observations has been used in the subsequent figures and tables. The magnetic fields were measured ballistically by comparison with a standard solenoid. For the greater part of the work the temperature gradient in the plate was about $25^{\circ} \mathrm{C}$. per centimeter, but in a few cases it had to be somewhat greater or less than $25^{\circ} \mathrm{C}$. per centimeter. The mean of the temperatures of the ends of the plate was taken as the temperature of the middle of the plate where the difference of potential was observed.

\section{RESULTS.}

The results of these observations on the nickel plate from Eimer and Amend have been plotted in Fig. 2 and those on the plate of Kahlbaum nickel in Fig. 3. The abscissæ in these figures are the magnetic fields in absolute units and ordinates are the corresponding differences of potential reduced to the case of a plate which is $\mathrm{I} \mathrm{cm}$. wide and has a temperature gradient of $\mathrm{I}^{\circ} \mathrm{C}$. per $\mathrm{cm}$. in it. From an examination of these figures it is seen that at the lower temperatures the electromotive force is at first proportional to the magnetic field, until the field reaches a value of about 5,000 absolute units. Above such fields a large increase in the magnetic field is accompanied by only small increases in the corresponding difference of potential. With this increase of the magnetic field the Nernst electromotive force seems to approach a limiting value just as the intensity of magnetization approaches a limiting value with an increase of the magnetizing force. As the temperature is increased the fields at which proportionality between the magnetic field and the Nernst electromotive force ceases, become smaller, so that the bends in the curves in Figs. 2 and 3 shift toward the left. It will be noticed from these curves that the fields for this saturated condition are somewhat larger for the plate of Eimer and Amend nickel than for the plate of Kahlbaum nickel. For the same temperature and equal magnetic fields the Nernst electromotive force is in general greater in the nickel plate from Eimer and Amend than in the nickel plate from Kahlbaum. When a temperature of about $35^{\circ} \mathrm{C}$. has been reached, the electromotive force for low fields is still increasing with rise of temperature but the electromotive force for high fields where the saturated condition is being reached is less than the corresponding electromotive force for a temperature of about $300^{\circ} \mathrm{C}$. At about $370^{\circ} \mathrm{C}$. for the plate of nickel from Eimer and 
[VoL. XXXIII.

Amend and at about $380^{\circ} \mathrm{C}$. for the plate of Kahlbaum nickel the character of the curve relating the Nernst electromotive force and the mag-

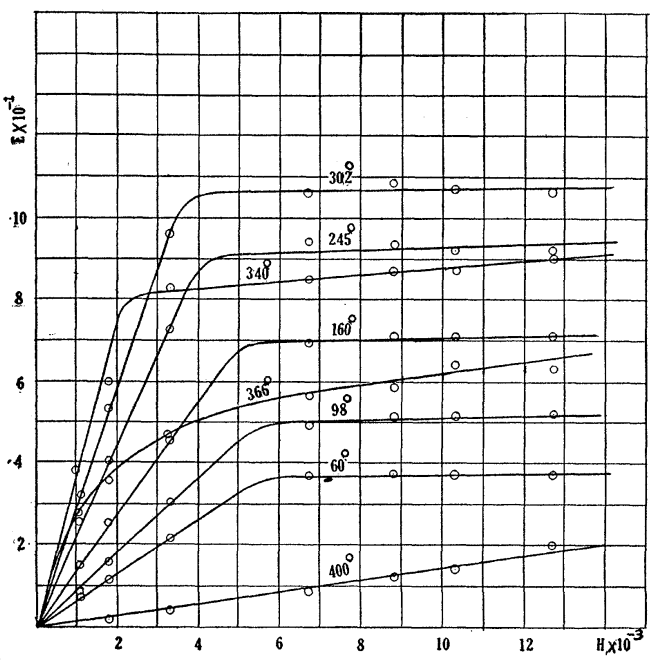

Fig. 2.

Nickel from Eimer and Amend. netic field has completely changed. There is still evidence of an approach to the saturated condition but it is not possible to reach that condition with the fields available in these experiments. Above $400^{\circ} \mathrm{C}$. the nickel has lost its magnetic properties and the Nernst electromotive force seems to be proportional to the magnetic fields over the range of fields used in these experiments. The magnitude of the effect decreases rapidly for small increases of temperature above the critical temperature, so that at about $400^{\circ} \mathrm{C}$. for a magnetic field of about 2,500 absolute units the Nernst electromotive force is only about one twentieth of the corresponding electromotiev force at a temperaturslightly less than the crite ical temperature.

The curves given in Figs. 2 and 3 are very similar in character to the corresponding curves relating the Hall electromotive force and the magnetic field. The reason for the shift in the bends in these curves toward lower fields is the same as the reason assigned for the correspond-

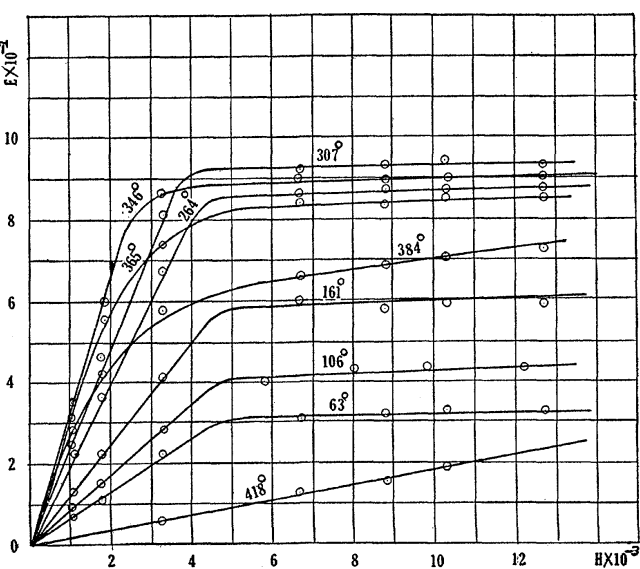

Fig. 3.

Kahlbaum Nickel. ing shift in the bends in the curves for the Hall effect. Both the Hall electromotive force and the Nernst electromotive force seem to be proportional to the intensity of magnetization in the plate rather than to the 
magnetic field, so that when the intensity of magnetization has reached its maximum value the Hall electromotive force and the Nernst electromotive force will reach a maximum value. In nickel the maximum value of the intensity of magnetization decreases continuously with the rise of temperature, at first slowly and then more more rapidly, as the critical temperature is approached. Hence the higher the temperature the lower the field in the air-gap necessary to produce saturation in the plate.

In Fig. 4 the value of $Q$ in the equation $E=Q \beta H \partial T / \partial x$ has been plotted against the absolute temperature of the plate. The values are

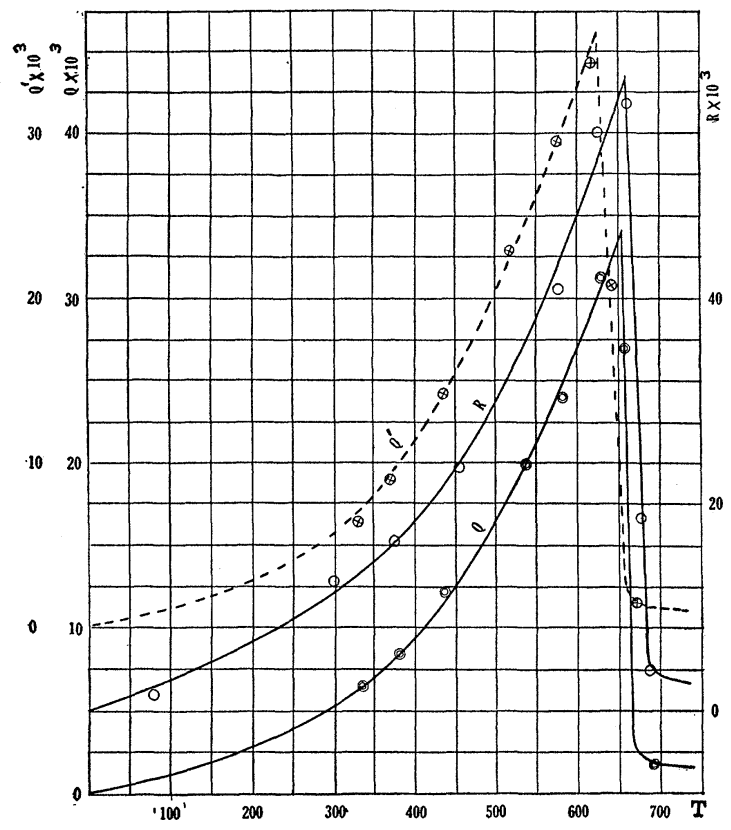

Fig. 4.

Nickel.

plotted for the region where the preceding curves showed that there was a proportionality between the Nernst electromotive force and the magnetic field. The lower curve is for the plate of Kahlbaum nickel; the upper curve for the plate of nickel from Eimer and Amend; the middle curve is taken from the previous paper and shows the relation between the Hall constant $R$ and the absolute temperature. The ordinates on the right refer to the Hall effect; those on the left to the Nernst effect. $Q$ means the constant for the Kahlbaum nickel plate and $Q^{\prime}$ the constant for the plate of Eimer and Amend nickel. The curves have been displaced vertically in order to avoid superposition. It is seen that the 
[Vol. XXXIII.

character of the curve is the same whether it is for the Hall effect or for the Nernst effect. The sudden decrease in the Hall effect and the corresponding decrease in the Nernst effect occur at nearly the same temperature. The curve for $Q$ as well as that for $Q^{\prime}$ has been extended back to the absolute zero.

Table I. contains the values of $Q, Q^{\prime}$ and $R$ read off of the curves in Fig. 4 at intervals of fifty degrees between $300^{\circ}$ and $650^{\circ}$ absolute. In the last column of this table the ratio of $Q$ to $R$ has been tabulated, and in Fig. 7 this ratio is plotted against the absolute temperature. From this ratio it is seen that $Q$ increases more rapidly than $R$ with increase of temperature. The comparison between the values of $Q^{\prime}$ and $R$ is less certain because the plate in which $Q^{\prime}$ was determined and that in which $R$ was determined certainly differed somewhat in purity. The results on the nickel plate from Eimer and Amend, however, confirm the results on the Kahlbaum nickel plate. At $32^{\circ} \mathrm{C}$. the value of the Nernst effect is nearly the same in the two plates. With rising temperature it increases somewhat more rapidly in the Eimer and Amend plate than in the Kahlbaum plate, but at their respective critical temperatures the magnitude of the effect in both plates is about the same. The critical temperature in the former is reached earlier than in the latter. As the critical temperature is approached the ratio of $Q$ to $R$ approaches a constant value. Nernst ${ }^{1}$ found $Q$ to have a value of $8.0 \mathrm{r} \times \mathrm{Io}^{-3}$ at $56^{\circ} \mathrm{C}$. when the magnetic field was about 830. At about the same temperature $Z \mathrm{Zahn}^{2}$ gives for one plate of nickel $Q=\mathrm{I} .3 \times 1 \mathrm{I}^{-3}$ when the magnetic field is 10,620; for another plate of nickel he gives for $Q=$ $3.55 \times \mathrm{IO}^{-3}$ when the magnetic field is 6,290 . The value given in this paper is larger than the value given by Zahn and less than that given by Nernst. This lack of agreement between the values obtained by different observers may be attributed to impurities in the nickel.

TABLE I.

Nickel.

\begin{tabular}{c|c|c|c|c}
\hline Abs. Temp. & $R \times \mathrm{ro}^{3}$ & $Q \times \mathrm{ro}^{3}$ & $Q^{\prime} \times \mathrm{ro}^{3}$ & $Q \mid R \times 1 \mathrm{ro}$ \\
\hline $300^{\circ}$ & 11.2 & 5.25 & 5.25 & 4.69 \\
350 & 14.8 & 7.25 & 7.7 & 4.90 \\
400 & 18.8 & 9.75 & 11.3 & 5.19 \\
450 & 24.0 & 13.0 & 16.0 & 5.42 \\
500 & 30.2 & 16.7 & 21.2 & 5.53 \\
550 & 37.6 & 21.3 & 26.2 & 5.67 \\
600 & 46.8 & 27.0 & 32.5 & 5.77 \\
650 & 59.0 & 34.0 & - & 5.76 \\
\hline
\end{tabular}

${ }^{1}$ W. A., 3I, p. $760,1887$.

2 Ibid., p. 92 I. 
Fig. 5 shows the relation between the Nernst electromotive force in cobalt and the magnetic field. The electromotive force is approximately proportional to the magnetic field over the range of fields used in these experiments. At $56^{\circ} \mathrm{C}$. there is a suggestion that the saturated condition is being reached but at higher temperatures it is not possible to reach saturation with the magnetic fields here available. In the work on the Hall effect it was seen that between $-190^{\circ} \mathrm{C}$. anda bout $300^{\circ} \mathrm{C}$. the fields necessary to obtain saturation increased with rising temperature and for higher temperatures there is a corresponding decrease with rising temperature. The field for which saturation begins to appear at $56^{\circ} \mathrm{C}$.

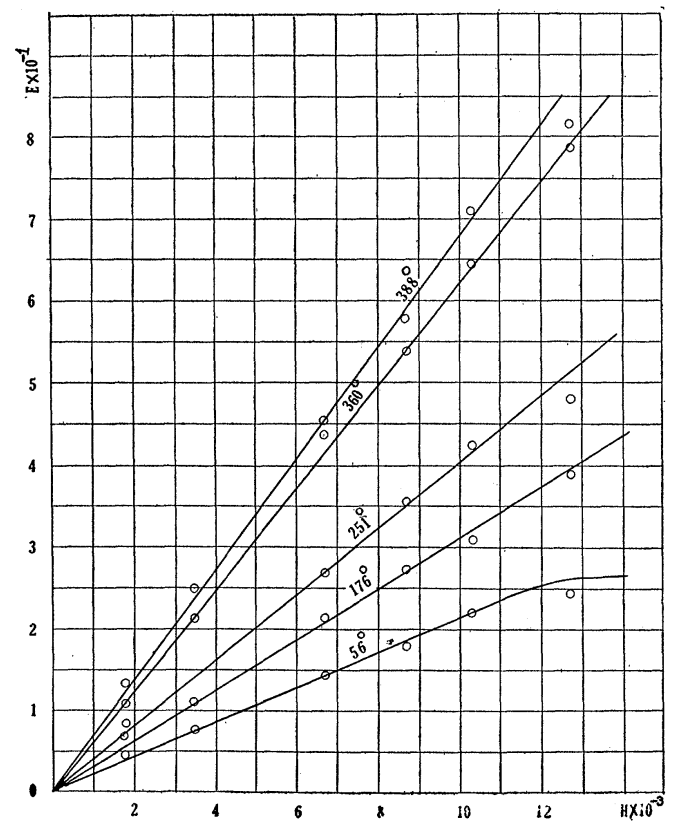

Fig. 5.

Cobalt. in the Nernst effect seems to be nearly equal to the field at which saturation begins to appear in the Hall effect at the same temperature. The shift toward higher fields agrees with the corresponding shift in the Hall effect. The explanation of this shift is the same as that given for the analogous shift in nickel.

The curves in Fig. 6 show the relation between $Q$ and the temperature and the relation between $R$ and the temperature in cobalt. The abscissæ are temperatures on the absolute scale; the ordinates on the right are for $R$ and those on the left are for $Q$. The character of the two curves is essentially the same but $R$ increases a little more rapidly than $Q$, so that the ratio of $Q / R$ decreases with rising temperature. This decrease becomes less rapid at higher temperatures and apparently the ratio approaches a constant value. The values of $Q$ and of $R$ have been read off of these curves at intervals of fifty degrees and the values tabulated in Table II. The last column of that table contains the ratio $Q / R$. In Fig. 7 this ratio has been plotted against the absolute temperature. Zahn gives for the value of $Q$ in cobalt $1.8 \times 10^{-3}$; Nernst found for it 
the value $2.24 \times \mathrm{IO}^{-3}$. The value $\mathrm{I} .8 \mathrm{o} \times \mathrm{IO}^{-3}$ for the plate studied in this paper agrees well with the value given by Zahn.

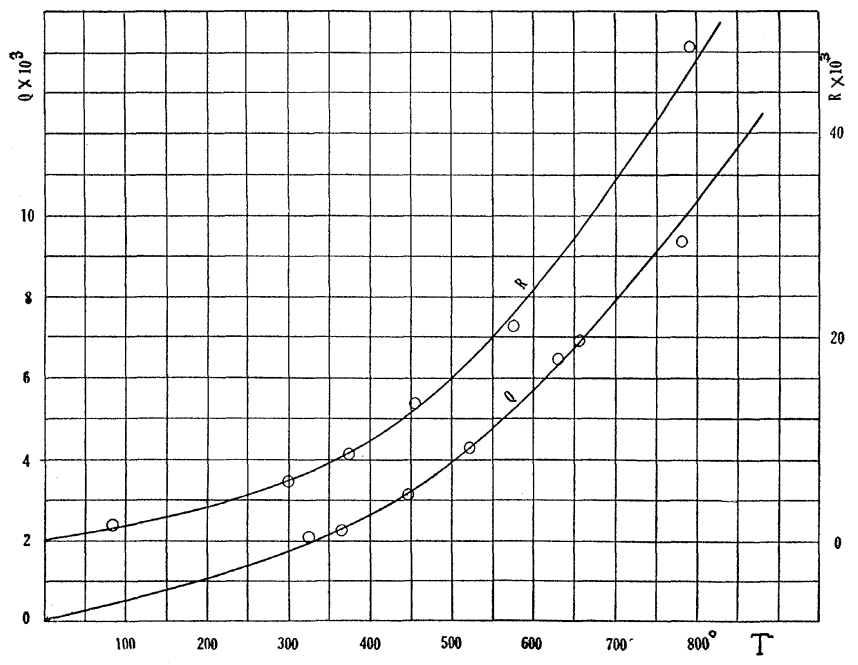

Fig. 6.

Cobalt.

In Fig. 7 besides the curves showing the relation in nickel and cobalt between $Q / R$ and the temperature a similar curve has been plotted for

TABLE II.

Cobalt.

\begin{tabular}{c|c|c|c}
\hline Abs. Temp. & $R \times \mathrm{ro}^{3}$ & $Q \times \mathrm{ro}^{3}$ & $Q / R \times$ 10 \\
\hline $300^{\circ}$ & 5.88 & 1.80 & 3.00 \\
350 & 7.60 & 2.15 & 2.83 \\
400 & 10.0 & 2.70 & 2.70 \\
450 & 12.6 & 3.30 & 2.62 \\
500 & 16.0 & 4.00 & 2.50 \\
550 & 20.0 & 4.85 & 2.42 \\
600 & 24.6 & 5.70 & 2.32 \\
650 & 29.6 & 6.75 & 2.28 \\
700 & 35.0 & 7.80 & 2.23 \\
750 & 40.8 & 8.90 & 2.18 \\
800 & 46.8 & 10.00 & 2.16 \\
\hline
\end{tabular}

soft iron from the data of Hall and Campbell. ${ }^{1}$ In nickel the least magnetic of these substances $Q$ increases more rapidly than $R$; in cobalt which is more magnetic than nickel but less mangetic than iron $Q$ in-

1 Ibid., p. 644. 
creases somewhat less rapidly than $R$; and in iron $Q$ increases much less rapidly than $R$. It seems that the more magnetic the substance the

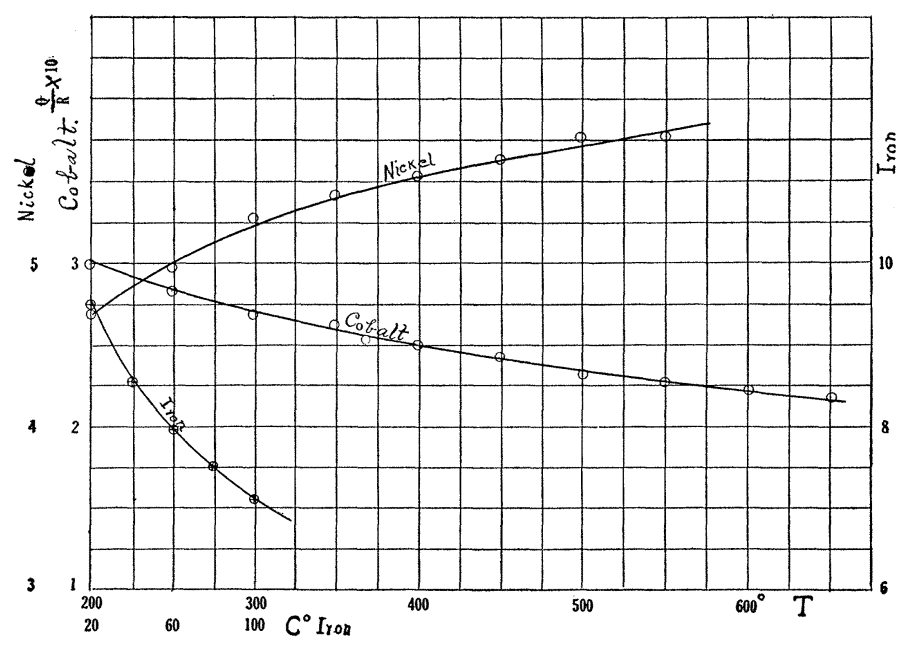

Fig. 7.

more rapid will be the decrease of $Q / R$ with rising temperature and that for a metal no more magnetic than nickel this ratio will increase.

The behavior of the Hall effect and the Nernst effect where the molecular transformation from $\alpha$-nickel to $\beta$-nickel takes place is of interest in connection with the relation between these effects and the resistance and the thermoelectric heights of the metals. Baedeker ${ }^{1}$ has called attention to the fact that an increase of resistance is accompanied by an increase in the Hall effect. The author's ${ }^{2}$ observations on the Hall effect in alloys points to the same conclusion, but they also make evident that there is not a proportionality between the Hall effect and the resistance in alloys. The data here given together with the data by Harrison ${ }^{3}$ on the resistance of nickel shows that the resistance depends on the temperature in a different way from the way in which the Hall effect and the Nernst effect depend upon it. At the critical temperature there is a small decrease in the rate at which the resistance increases with the temperature but there is a marked decrease in the Hall effect and in the Nernst effect. The factors which determine the changes in the former can only determine to a small degree the changes in the latter. A relation between the thermoelectric heights and the Hall effect has been previously pointed out. The

${ }^{1}$ Electr. Erscheinungen in Metallischen Leitern, p. ro4.

2 Phys. Rev., 32, p. I78, rori.

${ }^{3}$ Phil. Mag. (6), 3, p. 177. 
metals which have the largest thermoelectric height with respect to lead have in general the larger Hall effects. The observations of Harrison on the thermoelectric heights of nickel and those of Cernak ${ }^{1}$ on the Peltier effect in nickel show in each of these phenomena only small changes at the critical temperature. Neither the Hall effect nor the Nernst effect can be therefore regarded as determined completely by the factors which determine the thermoelectric heights and the Peltier effect.

\section{Summary.}

The chief results of this paper are:

I. The relation between the Nernst electromotive force and the magnetic field has been examined in nickel at a number of temperatures between $56^{\circ}$ and $410^{\circ} \mathrm{C}$. For a given magnetic field it has been found to increase with rising temperature until the critical temperature has been reached. The rate of increase becomes greater the nearer the critical temperature is approached. In passing the critical temperature the Nernst effect sinks to a small fraction of its value at that temperature and then probably decreases with further rise of temperature.

2. For any particular temperature below the critical temperature the Nernst electromotive force is at first proportional to the magnetic field but when the intensity of magnetization approaches its maximum there is a rapid deviation from this proportionality and the electromotive force approaches a limiting value at higher magnetic fields. Since the maximum intensity of magnetization decreases with rising temperature, the fields necessary to produce saturation become less as the temperature is increased.

3. At temperatures above the critical temperature the Nernst electromotive force is proportional to the magnetic field.

4. The study of the Nernst effect in cobalt between $56^{\circ}$ and $55^{\circ}$ has showed that the Nernst electromotive force is proportional to the magnetic field over that interval of temperature and over the range of magnetic fields in these experiments.

5. In nickel the Nernst effect increases more rapidly than the Hall effect with rising temperature and in cobalt it increases less rapidly than the Hall effect. In this respect cobalt behaves like iron.

1 Ann. der Phys., 24, p. 351, I907.

Physical Laboratory, Ohio State University. 